\title{
On Extended Convex Functions via Incomplete Gamma Functions
}

\author{
Yan Zhao $\mathbb{D}^{1}{ }^{1}$ M. Shoaib Saleem, ${ }^{2}$ Shahid Mehmood, ${ }^{2}$ and Zabidin Salleh $\mathbb{D}^{3}$ \\ ${ }^{1}$ School of Medical Engineering, Xinxiang Medical University, Xinxiang, Henan 453003, China \\ ${ }^{2}$ Department of Mathematics, University of Okara, Okara, Pakistan \\ ${ }^{3}$ Department of Mathematics, Faculty of Ocean Engineering Technology and Informatics, Universiti Malaysia Terengganu, \\ 21030 Kuala Nerus, Terengganu, Malaysia
}

Correspondence should be addressed to Zabidin Salleh; zabidin@umt.edu.my

Received 19 June 2021; Accepted 13 July 2021; Published 31 July 2021

Academic Editor: Sibel Yalçın

Copyright (C) 2021 Yan Zhao et al. This is an open access article distributed under the Creative Commons Attribution License, which permits unrestricted use, distribution, and reproduction in any medium, provided the original work is properly cited.

Convex functions play an important role in many areas of mathematics. They are especially important in the study of optimization problems where they are distinguished by a number of convenient properties. In this paper, firstly we introduce the notion of $h$-exponential convex functions. This notion can be considered as generalizations of many existing definitions of convex functions. Then, we establish some well-known inequalities for the proposed notion via incomplete gamma functions. Precisely speaking, we established trapezoidal, midpoint, and He's inequalities for $h$-exponential and harmonically exponential convex functions via incomplete gamma functions. Moreover, we gave several remarks to prove that our results are more generalized than the existing results in the literature.

\section{Introduction}

Convex optimization contributed largely in many areas of pure and applied mathematics during recent years, and convex analysis provides main foundation for convex optimization $[1,2]$. Due to huge applications of convex analysis, the researchers always show interest to generalization the notion of convexity. In literature, there exist many versions of convex functions, for example, $h$-convex function, see [3], $r$-convex functions, see [4], harmonic convex function, see [5], exponentially convex functions, see [6], etc. [7, 8].

Since convex function is a class of very important functions which is widely used in pure mathematics, functional analysis, optimization theory, and mathematical economics, so to study properties of certain classes of convex functions and establish different inequalities like trapezoidal, midpoint, He's Hermite-Hadamard, Fejér, etc., type inequality is an important area of research. A lot of work is devoted to establish different kinds of inequalities for different classes of convex functions, for example, Iscan [9] established Hermite-Hadamard type inequalities for harmonically convex functions. Bai et al. [10] presented Hermite-Hadamard type inequalities for the $m$ and $(\alpha, m)$-logarithmically convex functions. Özdemir et al. [11] developed HermiteHadamard-type inequalities via $(\alpha, m)$-convex functions. Chu et al. [12] gave generalizations of Hermite-Hadamard type inequalities for MT-convex functions.

It is always appreciable to derive more version of inequalities for generalized convexities. For some important generalization, we refer $[13,14]$. Fractional calculus also provides some broader variety to deal real-world problems. Just like other fields, fractional calculus also sets new trends in inequalities of convex analysis. For more details on fractional integral inequalities, we refer to the readers [15-18]. Many interesting controversies are also part of history of fractional calculus. Some famous definitions of fractional derivative are Riemann-Liouville [19], Caputo-Fabrizio [20], etc. [21-24]. In the present paper, we will deal with incomplete gamma functions. Firstly, we introduce the notions of $h$-exponential convex functions and harmonically exponential convex functions. Then, we establish some well-known inequalities for the proposed notions via incomplete gamma functions. Precisely speaking, we established trapezoidal, midpoint, and He's inequalities for $h$-exponential and harmonically 
exponential convex functions via incomplete gamma functions. Moreover, we gave several remarks to prove that our results are more generalized than the existing results in the literature.

The breakup of this paper is as follows: In Section 2, we present basic definitions and known results. Section 3 contains trapezoidal type inequalities via incomplete gamma function. Midpoint inequalities via incomplete gamma function are presented in Section 4, and He's inequality via the incomplete gamma functions is presented in Section 5. Last section contains concluding remarks and some future directions.

\section{Preliminaries}

Before starting the main findings, we review some definitions, notations, and theorems which are necessary to proceed. Throughout this paper, $L^{1}$ denotes space of all locally integrable functions.

Definition 1 [19]. For any $L^{1}$ function $z(u)$ on an interval $[x, y]$ with $u \in[x, y] k$-th left-RL fractional integral of $z(u)$ is given by

$$
\mathrm{RL}_{a^{+}}^{k} z(u)=\frac{1}{\Gamma(k)} \int_{x}^{u}(u-t)^{k-1} z(t) d t
$$

for $\operatorname{Re}(k)>0$. Also, the $k$-th right- $\mathrm{RL}$ fractional integral of $z(u)$ is given by

$$
{ }^{R L} J_{x}^{k} z(u)=\frac{1}{\Gamma(k)} \int_{u}^{y}(t-u)^{k-1} z(t) d t
$$

Definition 2 [6]. We say that the function $z: M \subseteq R \longrightarrow R$ is exponential type convex on $M$ if

$$
z(t x+(1-t) y) \leq\left(e^{t}-1\right) z(x)+\left(e^{1-t}-1\right) z(y)
$$

holds for every $x, y \in M$ and $t \in[0,1]$.

Definition 3 [3]. We say that the function $z: M \subseteq R \longrightarrow$ is $h$ convex function on $M$ if

$$
z(t x+(1-t) y) \leq h(t) z(x)+h(1-t) z(y)
$$

where $x, y \in M$ and $t \in[0,1]$.

We are now ready to define some new convexity, called as $h$-exponential convex function.

Definition 4. We say that the function $z: M \subseteq R \longrightarrow$ is $h$ exponential type convex on $M$ if

$$
z(t x+(1-t) y) \leq h\left(e^{t}-1\right) z(x)+h\left(e^{1-t}-1\right) z(y)
$$

where $x, y \in M$ and $t \in[0,1]$.
Remark 5.

(1) By substituting $h\left(e^{t}-1\right)=1 /\left(e^{t}-1\right), h\left(e^{1-t}-1\right)=$ $1 /\left(e^{1-t}-1\right.$ in Definition 3 , we get harmonically exponential convex function

(2) By substituting $h\left(e^{t}-1\right)=e^{t}-1, h\left(e^{1-t}-1\right)=e^{1-t}-1$ in Definition 3, we get Definition 2 of exponential convex function

Now, the integral inequality of Hermite-Hadamard (HH) type for a convex function is give by

$$
z\left(\frac{x+y}{2}\right) \leq \frac{1}{y-x} \int_{x}^{y} z(x) d x \leq \frac{z(x)+z(y)}{2} .
$$

Sarikaya et al. [25] generalized the HH-inequality (6) to fractional integrals of RL type which is given by

$$
z\left(\frac{x+y}{2}\right) \leq \frac{\Gamma(k+1)}{2(y-x)^{k}}\left[{ }^{R L} J_{x^{+}}^{k} z(y)+{ }^{R L} J_{y^{-}}^{k} z(x)\right] \leq \frac{z(x)+z(y)}{2},
$$

where $k>0$ and $z[x, y] \longrightarrow R$ is let to be an $L^{1}$ convex function. After that, Sarikaya and Yildirim [26] found a new inequality of the above

$$
\begin{aligned}
z\left(\frac{x+y}{2}\right) & \leq \frac{2^{k-1} \Gamma(k+1)}{(y-x)^{k}}\left[{ }^{\mathrm{RL}} J_{\left(\frac{x+y}{2}\right)}^{k} z(y)+{ }^{R L} J_{\left(\frac{x+y}{2}\right)}^{k} z(x)\right] \\
& \leq \frac{z(x+y)}{2}
\end{aligned}
$$

The following facts will be needed in establishing our main results:

Remark 6 (21). For $\operatorname{Re}>0$, the following identities hold:

$$
\begin{aligned}
\int_{0}^{1} t^{k-1} e^{t} d t & =(-1)^{k} \gamma(k,-1) ; \\
\int_{0}^{1} t^{k-1} e^{1-t} d t & =\gamma(k, 1), \\
\gamma(k, x) & =\int_{0}^{x} t^{k-1} e^{-t} d t, x \in C .
\end{aligned}
$$

Remark 7 (21). For $\operatorname{Re}>0$, the following identities hold:

$$
\begin{aligned}
\int_{0}^{1} t^{k-1} e^{t / 2} d t & =(-2)^{k} \gamma\left(k, \frac{-1}{2}\right) ; \\
\int_{0}^{1} t^{k-1} e^{1-(t / 2)} d t & =e 2^{k} \gamma\left(k, \frac{1}{2}\right) .
\end{aligned}
$$


Lemma 8 [25]. If $z:[x, y] \longrightarrow R$ is $L^{1}[x, y]$ with $0<x<y$ and $k>0$, then we have

$$
\begin{gathered}
\frac{z(x)+z(y)}{2}-\frac{\Gamma(k+1)}{(y-x)^{k}}\left[{ }^{R L} J_{x^{+}} z(y)+{ }^{R L} J_{y^{-}}^{k} z(x)\right. \\
=\frac{y-x}{2} \int_{0}^{1}\left[(1-t)^{k}-t^{k}\right] z(t x+(1-t) y) d t .
\end{gathered}
$$

Lemma 9 [26]. If $z:[x, y] \longrightarrow R$ is $L^{1}[x, y]$ with $0<x<y$ and $k>0$, then we have

$$
\begin{aligned}
& \frac{2^{k-1} \Gamma(k+1)}{(y-x)^{k}}\left[{ }^{R L} J_{((x+y) / 2)^{k}}^{k} z(y)+{ }^{R L} J_{((x+y) / 2)^{-}}^{k} z(x)-z\left(\frac{x+y}{2}\right)\right] \\
& \quad=\frac{y-x}{4}\left[\int_{0}^{1} e^{t} z\left(\frac{t}{2} x+\frac{2-t}{2} y\right) d t-\int_{0}^{1} t^{k} z\left(\frac{2-t}{2} x+\frac{t}{2} y\right) d t\right] .
\end{aligned}
$$

\section{Trapezoidal Type Inequalities via Incomplete Gamma Function}

In this section, we present trapezoidal type inequalities via incomplete gamma function.

Theorem 10. Suppose that $z:[x, y] \longrightarrow R$ is $L^{1}[x, y]$ and $h$ exponential convex function, then we have for $k>0$,

$$
\begin{aligned}
z\left(\frac{x+y}{2}\right) & \leq \frac{k h\left(e^{1 / 2}-1\right) \Gamma(k+1)}{(y-x)^{k}}\left[{ }^{R L} J_{x}^{k} z(y)+{ }^{R L} J_{y}^{k} z(x)\right] \\
& \leq h\left(e^{1 / 2}-1\right) M[z(x)+z(y)]
\end{aligned}
$$

where $h\left(e^{t}+e^{1-t}-2\right) \leq M$.

Proof. Let $z: I \longrightarrow R$ is $h$-exp convex function and $k>0$ then by definition

$$
\begin{aligned}
z\left(\frac{x+y}{2}\right)= & z\left[\frac{(t x+(1-t) y)+(1-t) x+t y}{2}\right] \\
\leq & h\left(e^{1 / 2}-1\right) z(t x+(1-t) y) \\
& \left.+h\left(e^{1 / 2}-1\right) z((1-t) x+t y)\right) .
\end{aligned}
$$

Multiplying $t^{k-1}$ on both sides and then integrating on $[0,1]$, we get

$$
\begin{aligned}
\frac{1}{k} z\left(\frac{x+y}{2}\right) \leq & \left.h\left(e^{1 / 2}-1\right) \int_{0}^{1} t^{k-1} z(t x+(1-t) y)\right) d t \\
& \left.+h\left(e^{1 / 2}-1\right) \times \int_{0}^{1} t^{k-1} z((1-t) x+t y)\right) d t .
\end{aligned}
$$

Again by small substitution, we have

$$
\begin{aligned}
z\left(\frac{x+y}{2}\right) \leq & k h\left(e^{(1 / 2)-1}\right) \frac{1}{x-y}\left[\int_{y}^{x}\left(\frac{y-u}{y-x}\right)^{k-1} z(u) d(u)\right. \\
& \left.+\int_{x}^{y}\left(\frac{v-x^{k-1}}{y-x}\right) z(v) d(v)\right] \\
\leq & k h\left(e^{1 / 2}-1\right)\left[\frac{1}{x-y} \int_{y}^{x}\left(\frac{y-u}{y-x}\right)^{k-1} z(u) d(u)\right. \\
& \left.+\frac{1}{x-y} \int_{x}^{y}\left(\frac{v-x}{y-x}\right)^{k-1} z(v) d(v)\right] \\
\leq & \frac{\Gamma(k+1) h\left(e^{1 / 2}-1\right)}{(x-y)^{k}}\left[{ }^{\mathrm{RL}} J_{x^{+}}^{k} z(y)+{ }^{\mathrm{RL}} J_{y^{-}}^{k} z(x)\right] .
\end{aligned}
$$

For other inequalities, take

$$
\begin{aligned}
& z(t x+(1-t) y) \leq h\left(e^{t}-1\right) z(x)+h\left(e^{1-t}-1\right) z(y) \\
& z((1-t) x+t y) \leq h\left(e^{1-t}-1\right) z(x)+h\left(e^{t}-1\right) z(y) .
\end{aligned}
$$

Adding both inequalities, we get

$$
z(t x+(1-t) y)+z((1-t) x+t y) \leq h\left(e^{t}+e^{1-t}-2\right)[z(x)+z(y)] .
$$

Multiplying both sides by $t^{k-1}$ and integrating on $[0,1]$, we have

$$
\begin{aligned}
& \int_{0}^{1} t^{k-1} z(t x+(1-t) y) d t+\int_{0}^{1} t^{k-1} z((1-t) x+t y) d t \\
& \quad \leq \int_{0}^{1} t^{k-1} h\left(e^{t}+e^{1-t}-2\right) d t[z(x)+z(y)] .
\end{aligned}
$$

By making the change of variables

$$
\begin{aligned}
& \frac{1}{x-y} \int_{y}^{x}\left(\frac{y-u}{y-x}\right)^{k-1} z(u) d(u)+\frac{1}{x-y} \int_{y}^{x}\left(\frac{v-x}{y-x}\right)^{k-1} z(v) d(v) \\
& \quad \leq[z(x)+z(y)] \int_{0}^{1} t^{k-1} h\left(e^{t}+e^{1-t}-2\right) d t, \\
& \frac{\Gamma(k)}{(x-y)^{k}}\left[\mathrm{RL}^{k} J_{x^{+}}^{k} z(y)+{ }^{\mathrm{RL}} J_{y^{-}}^{k} z(x)\right] \\
& \quad \leq[z(x)+z(y)] \int_{0}^{1} t^{k-1} h\left(e^{t}+e^{1-t}-2\right) d t .
\end{aligned}
$$


Multiplying by $k>0$ and $h\left(e^{1 / 2}-1\right)$ on both sides, we get.

$$
\begin{aligned}
& \frac{\Gamma(k+1) h\left(e^{1 / 2}-1\right)}{(x-y)^{k}}\left[{ }^{\mathrm{RL}} J_{x^{+}}^{k} z(y)+{ }^{\mathrm{RL}} J_{y^{-}}^{k} z(x)\right] \\
& \leq k h\left(e^{1 / 2}-1\right)[z(x)+z(y)] \int_{0}^{1} t^{k-1} h\left(e^{t}+t^{1-t}-2\right) d t \\
& \frac{\Gamma(k+1) h\left(e^{1 / 2}-1\right)}{(x-y)^{k}}\left[{ }^{\mathrm{RL}} J_{x^{+}}^{k} z(y)+{ }^{\mathrm{RL}} J_{y^{-}}^{k} z(x)\right] \\
& \leq h\left(e^{1 / 2}-1\right) M[z(x)+z(y)] .
\end{aligned}
$$

Corollary 11. If we substitute $h\left(e^{t}+e^{1-t}-2\right)=\left(e^{t}+e^{1-t}-2\right)$ in (22) and use Remark 6, then both of inequalities (17) and (22) become (7) of [27].

Remark 12. For $h\left(e^{t}+e^{1-t}-2\right)=1 /\left(e^{t}+e^{1-t}-2\right)$, (22) yields trapezoidal type inequalities via the incomplete gamma function for harmonically exponential convex function.

Theorem 13. Let $z:[x, y] \longrightarrow R$ be $L^{1}[x, y]$ with $0<x<y$ and $k>0$. If $|z|$ is an h-exp convex function, then we

$$
\begin{aligned}
z\left(\frac{x+y}{2}\right) \leq & \frac{h\left(e^{1 / 2}-1\right) \Gamma(k+1)}{(x-y)^{k}}\left[R J_{x^{+}}^{k} z(y)+{ }^{R L} J_{y^{-}}^{k} z(x)\right] \\
\leq & \frac{y-x}{2}\left(\left[\delta_{0}\left(k, h_{0}, h\right)\right]+\delta_{1}\left(k, h_{1}, h\right)\right]|z(x)| \\
& +\left[\delta_{0}\left(k, h_{1}, h\right)+\delta_{1}\left(k, h_{0}, h\right)\right]|z(y)| \\
& +\left[\delta_{0}\left(k, h_{0}, h\right)+\delta_{0}\left(k, h_{1}, h\right)\right]|z(x)| \\
& +\left[\delta_{1}\left(k, h_{0}, h\right)+\delta_{0}\left(k, h_{0}, h\right)\right]|z(y)|
\end{aligned}
$$

where

$$
\left\{\begin{array}{l}
\delta_{0}\left(k \cdot h_{0}, h\right)=\int_{0}^{\frac{1}{2}}(1-t)^{k} h\left(e^{t}-1\right) d t=\int_{\frac{1}{2}}^{1} t^{k} h\left(e^{1-t}-1\right) d t \\
\delta_{0}\left(k, h_{1}, h\right)=\int_{0}^{\frac{1}{2}}(1-t)^{k} h\left(e^{1-t}-1\right) d t=\int_{\frac{1}{2}}^{1} t^{k} h\left(e^{t}-1\right) d t \\
\delta_{1}\left(k, h_{0}, h\right)=-\int_{0}^{\frac{1}{2}} t^{k} h\left(e^{1-t}-1\right) d t=-\int_{\frac{1}{2}}^{1}(1-t)^{k} h\left(e^{t}-1\right) d t \\
\delta_{1}\left(k, h_{1}, h\right)=-\int_{0}^{\frac{1}{2}} t^{k} h\left(e^{t}-1\right) d t=-\int_{\frac{1}{2}}^{1}(1-t)^{k} h\left(e^{1-t}-1\right) d t .
\end{array}\right.
$$

Proof. From Lemma 8, we have

$$
\begin{gathered}
\left|\frac{z(x)+z(y)}{2}-\frac{\Gamma(k+1)}{2(y-x)^{k}}\left[{ }^{\mathrm{RL}} J_{x^{+}}^{k} z(y)+{ }^{\mathrm{RL}} J_{y^{-}}^{k} z(x)\right]\right| \\
\left.\leq \frac{y-x}{2} \int_{0}^{1}(1-t)^{k}-t^{k}\right) \mid z(t x+(1-t) y \mid d t \\
=\frac{y-x}{2}\left[\int_{0}^{\frac{1}{2}}\left((1-t)^{k}-t^{k}\right)|z(t x+(1-t)) y| d t\right] \\
+\left[\int_{\frac{1}{2}}^{1}\left(t^{k}-(1-t)^{k}\right)|z(t x+(1-t)) y| d t\right] .
\end{gathered}
$$

By using the $h$-exp convexity of $|z|$

$$
\begin{aligned}
& \left|\frac{z(x)+z(y)}{2}-\frac{\Gamma(k+1)}{2(y-x)^{k}}\left[{ }^{\mathrm{RL}} J_{x^{+}}^{k} z(y)+{ }^{\mathrm{RL}} J_{y^{-}}^{k} z(x)\right]\right| \\
& \leq \frac{y-x}{2}\left[\int _ { 0 } ^ { \frac { 1 } { 2 } } ( ( 1 - t ) ^ { k } - t ^ { k } ) \left[h\left(e^{t}-1\right)|z(x)|\right.\right. \\
& \left.+h\left(e^{1-t}-1\right)|z(y)|\right] d t \\
& \quad+\int_{\frac{1}{2}}^{1}\left(t^{k}-(1-t)^{k}\right)\left[h\left(e^{t}-1\right)|z(x)|\right. \\
& \left.\left.+h\left(e^{1-t}-1\right)|z(y)|\right] d t\right] .
\end{aligned}
$$

By using identities (25), we get required result.

\section{Midpoint Inequalities via Incomplete Gamma Function}

This section contains midpoint inequalities via incomplete gamma function.

Theorem 14. Let $z:[x, y] \longrightarrow R$ be $L^{1}[x, y]$ with $0<x<y$ and $k>0$. If $|z|$ is an $h$-exp convex function, then we

$$
\begin{aligned}
& z\left(\frac{x+y}{2}\right) \frac{h\left(e^{1 / 2}-1\right) \Gamma(k+1)}{(x-y)^{k}}\left[R J_{((x+y) / 2)^{+}}^{k} z(y)+{ }^{R L} J_{((x+y) / 2)^{-}}^{k} z(x)\right] \\
& \quad \leq h\left(e^{1 / 2}-1\right) M[z(x)+z(y)]
\end{aligned}
$$

where $h\left(e^{t / 2}+e^{1-(t / 2)}-2\right) \leq M$.

Proof. Let $z: I \longrightarrow R$ is $h$-exponential convex function and $k>0$, then by definition

$$
\begin{aligned}
z\left(\frac{x+y}{2}\right) & =z\left(\frac{[(t / 2) x+((2-t) / 2) y]+[((2-t) / 2) x+(t / 2) y]}{2}\right) \\
& \leq h\left(e^{1 / 2}-1\right) z\left(\frac{t}{2} x+\frac{2-t}{2} y\right)+h\left(e^{1 / 2}-1\right) z\left(\frac{2-t}{2} x+\frac{t}{2} y\right)
\end{aligned}
$$


Multiplying by $t^{k-1}$ on both sides and integrating w.r.t " $\mathrm{t}$ " from $[0,1]$, we get

$$
\begin{aligned}
\frac{1}{k} z\left(\frac{x+y}{2}\right) \leq & h\left(e^{1 / 2}-1\right) \int_{0}^{1} t^{k-1} z\left(\frac{t}{2} x+\left(1-\frac{t}{2}\right) y\right) d t \\
& +h\left(e^{1 / 2}-1\right) \int_{0}^{1} t^{k-1} z\left(\frac{2-t}{2} x+\frac{t}{2} y\right) d t .
\end{aligned}
$$

Again by small substitution, we have

$$
\begin{aligned}
\frac{1}{k} z\left(\frac{x+y}{2}\right) \leq & h\left(e^{1 / 2}-1\right) \frac{1}{x-y} \int_{y}^{\frac{x+y}{2}} 2^{k}\left(\frac{y-u}{y-x}\right)^{k-1} z(u) d(u) \\
& +h\left(e^{1 / 2}-1\right) \frac{1}{x-y} \int_{\frac{x+y}{2}}^{x} 2^{k}\left(\frac{v-x}{y-x}\right)^{k-1} z(v) d(v) \\
\leq & 2^{k} h\left(e^{1 / 2}-1\right) \frac{1}{x-y} \int_{y}^{\frac{x+y}{2}}\left(\frac{y-u}{y-x}\right)^{k-1} z(u) d(u) \\
& +2^{k} h\left(e^{1 / 2}-1\right) \frac{1}{x-y} \int_{\frac{x+y}{2}}^{x}\left(\frac{v-x}{y-x}\right)^{k-1} z(v) d(v)
\end{aligned}
$$

Implies

$$
\begin{aligned}
z\left(\frac{x+y}{2}\right)= & \frac{h\left(e^{1 / 2}-1\right) 2^{k} \Gamma(k+1)}{(x-y)^{k}} \\
& \cdot\left[{ }^{\mathrm{RL}} J_{((x+y) / 2)^{+}}^{k} z(y)+{ }^{\mathrm{RL}} J_{((x+y) / 2)^{-}}^{k} z(x)\right]
\end{aligned}
$$

For other inequalities, take

$$
\begin{aligned}
& z\left(\frac{t}{2} x+\frac{2-t}{2} y\right) \leq h\left(e^{t / 2}-1\right) z(x)+h\left(e^{1-(t / 2)}-1\right) z(y) \\
& z\left(\frac{2-t}{2} x+\frac{t}{2} y\right) \leq h\left(e^{(1-t) / 2}-1\right) z(x)+h\left(e^{t / 2}-1\right) z(y) .
\end{aligned}
$$

Adding both inequalities, we have

$$
\begin{aligned}
& \left(\frac{t}{2} x+\frac{2-t}{2} y\right)+z\left(\frac{2-t}{2} x+\frac{t}{2} y\right) z \\
& \quad \leq h\left(e^{t / 2}+e^{1-(t / 2)}-2\right)[z(x)+z(y)]
\end{aligned}
$$

Multiplying by $t^{k-1}$ on both sides and integrating on $[0,1]$, we get

$$
\begin{gathered}
\int_{0}^{1} t^{k-1} z\left(\frac{t}{2} x+\frac{2-t}{2} y\right) d t+\int_{0}^{1} t^{k-1} z\left(\frac{2-t}{2} x+\frac{t}{2} y\right) d t \\
\leq[z(x)+z(y)] \int_{0}^{1} t^{k-1} h\left(e^{t / 2}+e^{1-(t / 2)}-2\right) d t
\end{gathered}
$$

By making the change of variables, we get

$$
\begin{aligned}
& 2^{k}\left[\frac{1}{x-y} \int_{y}^{\frac{x+y}{2}}\left(\frac{y-u}{y-x}\right)^{k-1} z(u) d(u)+\frac{1}{x-y} \int_{\frac{x+y}{2}}^{x}\left(\frac{v-x}{y-x}\right)^{k-1} z(v) d(v)\right] \\
& \quad \leq[z(x)+z(y)] \int_{0}^{1} t^{k-1} h\left(e^{t / 2}+e^{1-(t / 2)}-2\right) d t, \\
& \frac{2^{k} \Gamma(k)}{(x-y)^{k}}\left[{ }^{\mathrm{RL}} J_{((x+y) / 2)^{+}}^{k} z(y)+{ }^{\mathrm{RL}} J_{((x+y) / 2)^{-}}^{k} z(x)\right] \\
& \quad \leq[z(x)+z(y)] \int_{0}^{1} t^{k-1} h\left(e^{t / 2}+e^{1-(t / 2)}-2\right) d t .
\end{aligned}
$$
have

Multiplying $k>0$ and $h\left(e^{1 / 2}-1\right)>0$ on both sides, we

$$
\begin{aligned}
& \frac{h\left(e^{1 / 2}-1\right) 2^{k} \Gamma(k+1)}{(x-y)^{k}}\left[{ }^{\mathrm{RL}} J_{((x+y) / 2)^{+}}^{k} z(y)+{ }^{\mathrm{RL}} J_{((x+y) / 2)^{-}}^{k} z(x)\right] \\
& \quad \leq k h\left(e^{1 / 2}-1\right)[z(x)+z(y)] \int_{0}^{1} t^{k-1} h\left(e^{t / 2}+e^{1-(t / 2)}-2\right) d t \\
& \quad \leq h\left(e^{1 / 2}-1\right) M[z(x)+z(y)] .
\end{aligned}
$$

Corollary 15. When we introduced $h\left(e^{1 / 2}+e^{1-(t / 2)}-2\right)=e^{t}+$ $e^{1-(t / 2)}-2$ in (37) using Remark 7 and rearrange both inequalities (32) and (37), we get (10) of [27].

Remark 16. For $h\left(e^{t / 2}+e^{1-(t / 2)}-2\right)=1 /\left(e^{t / 2}+e^{1-(t / 2)}-2\right)$, (37) yields midpoint type inequalities via the incomplete gamma function for harmonically exponential convex function.

\section{He's Inequality via the Incomplete Gamma Functions}

He's inequality via the incomplete gamma functions is presented in this section.

Definition 17. For any $L^{1}$ function $z$ on interval $[0, s]$, the $k$-th He's fractional derivative of $z(x)$ is defined by

$$
\begin{aligned}
D_{s}^{k} w(s) & =\frac{1}{\Gamma(n-k)} \frac{d^{n}}{d s^{n}} \int_{0}^{s}(t-s)^{n-k-1} z(t) d t \\
z\left(\frac{x+y}{2}\right) & =z\left(\frac{[t x+(1-t) y]+[(1-t) x+t y]}{2}\right) .
\end{aligned}
$$

By using $h$-exponential convex function

$$
\begin{aligned}
z\left(\frac{x+y}{2}\right) \leq & h\left(e^{1 / 2}-1\right) z((t x+(1-t) y) \\
& +h\left(e^{1 / 2}-1\right) z((1-t) x+t y) .
\end{aligned}
$$


Taking $x=0$ and $y>0$ for all $s \in(0,1)$ and multiplying by $(t-s)^{n-k-1} / \Gamma(n-k)$, we get

$$
\begin{aligned}
& \frac{1}{\Gamma(n-k)} z\left(\frac{y}{2}\right) \int_{0}^{s}(t-s)^{n-k-1} d t \\
& \leq \frac{h\left(e^{1 / 2}-1\right)}{\Gamma(n-k)}\left[\int_{0}^{s}\left((t-s)^{n-k-1} z(1-t) y\right) d t\right. \\
& \left.\quad+\int_{0}^{s}(t-s)^{n-k-1} z(t y) d t\right] \frac{(-1)^{n-k} s^{n-k}}{\Gamma(n-k)} z\left(\frac{y}{2}\right) \\
& \leq \frac{h\left(e^{1 / 2}-1\right)}{\Gamma(n-k)}\left[\int_{0}^{s}\left((t-s)^{n-k-1} z(1-t) y\right) d t\right. \\
& \left.\quad+\int_{0}^{s}(t-s)^{n-k-1} z(t y) d t\right] .
\end{aligned}
$$

Hence

$$
\begin{aligned}
\frac{(-1)^{n-k} s^{n-k}}{\Gamma(n-k)} z\left(\frac{y}{2}\right) \leq & \frac{h\left(e^{1 / 2}-1\right)}{\Gamma(n-k)}\left[\int_{0}^{s}\left((t-s)^{n-k-1} z(1-t) y\right) d t\right. \\
& \left.+\int_{0}^{s}(t-s)^{n-k-1} z(t y) d t\right] .
\end{aligned}
$$

After getting the $n$-th derivatives on both sides of (41) w.r.t to $s$ and using Definition 17, we get

$$
(-1)^{n-k} z\left(\frac{y}{2}\right) \leq\left[D_{s b}^{k} z(s b)+(-1)^{n-k} D_{(1-s) b}^{k} z((1-s) b)\right] .
$$

Remark 18. By putting $h\left(e^{1 / 2}-1\right)=\left(e^{1 / 2}-1\right)$ in $(41)$, we get He's inequality (14) of [27].

5.1. He's Inequality for Harmonically Exponential Convex Function. From Definition 17 and by using definition of $h$ exponential convex function, we have

$$
\begin{aligned}
z\left(\frac{x+y}{2}\right) \leq & h\left(e^{1 / 2}-1\right) z((t x+(1-t) y) \\
& +h\left(e^{1 / 2}-1\right) z((1-t) x+t y) .
\end{aligned}
$$

By harmonically exponential convex function, we have $z\left(\frac{x+y}{2}\right) \leq \frac{1}{e^{1 / 2}-1} z\left((t x+(1-t) y)+\frac{1}{e^{1 / 2}-1} z((1-t) x+t y)\right.$.

Taking $x=0$ and $y>0$ for all $s \in(0,1)$, multiplying by $(t-s)^{n-k-1} / \Gamma(n-k)$

$$
\begin{aligned}
& \frac{1}{\Gamma(n-k)} z\left(\frac{y}{2}\right) \int_{0}^{s}(t-s)^{n-k-1} d t \\
& \leq \frac{1}{\left(e^{1 / 2}-1\right) \Gamma(n-k)}\left[\int_{0}^{s}\left((t-s)^{n-k-1} z(1-t) y\right) d t\right. \\
& \left.\quad+\int_{0}^{s}(t-s)^{n-k-1} z(t y) d t\right] \frac{(-1)^{n-k} s^{n-k}}{\Gamma(n-k)} z\left(\frac{y}{2}\right) \\
& \leq \frac{1}{\left(e^{1 / 2}-1\right) \Gamma(n-k)}\left[\int_{0}^{s}\left((t-s)^{n-k-1} z(1-t) y\right) d t\right. \\
& \left.\quad+\int_{0}^{s}(t-s)^{n-k-1} z(t y) d t\right] .
\end{aligned}
$$

Hence

$$
\begin{aligned}
\frac{(-1)^{n-k} s^{n-k}}{\Gamma(n-k)} z\left(\frac{y}{2}\right) \leq & \frac{1}{\left(e^{1 / 2}-1\right) \Gamma(n-k)} \\
& \cdot\left[\int_{0}^{s}\left((t-s)^{n-k-1} z(1-t) y\right) d t\right. \\
& \left.+\int_{0}^{s}(t-s)^{n-k-1} z(t y) d t\right] .
\end{aligned}
$$

After getting the $n$-th derivatives on both sides of (41) w.r.t to $s$ and using Definition 17, we get

$$
(-1)^{n-k} z\left(\frac{y}{2}\right) \leq\left[D_{s b}^{k} z(s b)+(-1)^{n-k} D_{(1-s) b}^{k} z((1-s) b)\right]
$$

Remark 19. For $1 /\left(e^{1 / 2}-1\right)=h\left(e^{1 / 2}-1\right)$ in (47), we get (41). Again substitute $h\left(e^{1 / 2}-1\right)=e^{t}-1$ in (41), we get He's inequality (14) of [27].

\section{Conclusion}

The inequalities in analysis play a vital role to study qualitative properties of functions and solutions of differential equations; we develop various Hermite-Hadamard type inequalities, midpoint inequalities, and trapezoidal and He's inequalities forh-exponential convex functions with appropriate substitutions; we may obtain the inequalities for harmonicallyh-exponential convex functions. To establish same inequalities for $h$-exponential convex stochastic processes is an interesting problem.

\section{Data Availability}

There is no additional data required for the finding of results of this paper.

\section{Conflicts of Interest}

It is declared that authors have no competing interests.

\section{Authors' Contributions}

All authors have equal contribution in this article.

\section{Acknowledgments}

This research has been partially supported by the Center of Research and Innovation Management, Universiti Malaysia Terengganu.

\section{References}

[1] C. Niculescu and L. E. Persson, Convex Functions and Their Applications (pp. xvi+-255), Springer, New York, 2006.

[2] A. W. Roberts, "Convex Functions," in Handbook of convex geometry, pp. 1081-1104, Elsevier, North-Holland, 1993. 
[3] M. Z. Sarikaya, A. Saglam, and H. Yildirim, "On some Hadamard-type inequalities for h-convex functions," Journal of Mathematical Inequalities, vol. 2, no. 3, pp. 335-341, 2008.

[4] P. M. Gill, C. E. M. Pearce, and J. Pecaric, "Hadamard's inequality for r-convex functions," Journal of Mathematical Analysis and Applications, vol. 215, no. 2, pp. 461-470, 1997.

[5] I. Abbas Baloch and Y. M. Chu, "Petrović-type inequalities for Harmonic -convex functions," Journal of Function Spaces, vol. 2020, 7 pages, 2020.

[6] M. U. Awan, M. A. Noor, and K. I. Noor, "Hermite-Hadamard inequalities for exponentially convex functions," Applied Mathematics \& Information Sciences, vol. 12, no. 2, pp. 405409, 2018.

[7] M. U. Awan, M. A. Noor, M. V. Mihai, K. I. Noor, and N. Akhtar, "On approximately harmonic h-convex functions depending on a given function," Univerzitet u Nišu, vol. 33, no. 12, pp. 3783-3793, 2019.

[8] I. A. Baloch, S. S. Dragomir, and S. S. Dragomir, "New inequalities based on harmonic log-convex functions," Open Journal of Mathematical Analysis, vol. 3, no. 2, pp. 103-105, 2019.

[9] I. Iscan, "Hermite-Hadamard type inequalities for harmonically convex functions," Hacettepe Journal of Mathematics and statistics, vol. 43, no. 6, pp. 935-942, 2014.

[10] R. F. Bai, F. Qi, and B. Y. Xi, "Hermite-Hadamard type inequalities for the $\mathrm{m}$-and $(\alpha, \mathrm{m})$-logarithmically convex functions," Univerzitet u Nišu, vol. 27, no. 1, pp. 1-7, 2013.

[11] M. E. Özdemir, M. Avci, and H. Kavurmaci, "Hermite-Hadamard-type inequalities via $(\alpha, \mathrm{m})$ - convexity," Computers \& Mathematics with Applications, vol. 61, no. 9, pp. 2614-2620, 2011.

[12] Y. M. Chu, M. A. Khan, T. U. Khan, and T. Ali, "Generalizations of Hermite-Hadamard type inequalities for MT-convex functions," Journal of Nonlinear Sciences and Applications, vol. 9, no. 5, pp. 4305-4316, 2016.

[13] M. Kadakal and I. Iscan, "Exponential type convexity and some related inequalities," Journal of Inequalities and Applications, vol. 2020, Article ID 82, 2020.

[14] Y. Zhao, H. Sang, and W. Xiong, "Hermite-Hadamard-type inequalities involving the Riemann-Liouville fractional integrals via h-convex functions," Journal of inequalities and Applications, vol. 2020, 2020.

[15] Y. C. Kwun, G. Farid, W. Nazeer, S. Ullah, and S. M. Kang, "Generalized Riemann-Liouville k-fractional integrals associated with Ostrowski type inequalities and error bounds of hadamard inequalities," IEEE access, vol. 6, pp. 64946-64953, 2018.

[16] X. Z. Yang, G. Farid, W. Nazeer, Y. M. Chu, and C. F. Dong, "Fractional generalized Hadamard and Fejér-Hadamard inequalities for m-convex function," AIMS Math, vol. 5, no. 6, pp. 6325-6340, 2020.

[17] A. Adu-Sackey, F. T. Oduro, F. T. Oduro, and G. O. Fosu, "Inequalities approach in determination of convergence of recurrence sequences," Open Journal of Mathematical Sciences, vol. 5, no. 1, pp. 65-72, 2021.

[18] G. Farid, K. Mahreen, and Y. M. Chu, "Study of inequalities for unified integral operators of generalized convex functions," Open Journal of Mathematical Sciences, vol. 5, pp. 80-93, 2021.

[19] M. Riesz, "L'intégrale de Riemann-Liouville et le problème de Cauchy," Acta Mathematica, vol. 81, pp. 1-222, 1949.
[20] M. Caputo and M. Fabrizio, "On the notion of fractional derivative and applications to the hysteresis phenomena," Meccanica, vol. 52, no. 13, pp. 3043-3052, 2017.

[21] D. Baleanu, A. Kashuri, P. O. Mohammed, and B. Meftah, "General Raina fractional integral inequalities on coordinates of convex functions," Advances in Difference Equations, vol. 2021, Article ID 82, 2021.

[22] M. B. Khan, P. O. Mohammed, M. A. Noor, and Y. S. Hamed, "New Hermite-Hadamard inequalities in fuzzy-interval fractional calculus and related inequalities," Symmetry, vol. 13, no. 4 , p. $673,2021$.

[23] B. Meftah, A. Souahi, and A. Souahi, "Cebyšev inequalities for co-ordinated $\backslash(\mathrm{QC} \backslash)$-convex and $\backslash((\mathrm{s}, \mathrm{QC}) \backslash)$-convex," Engineering and Applied Science Letters, vol. 4, no. 1, pp. 14-20, 2021.

[24] G. Farid, A. U. Rehman, S. Bibi, and Y. M. Chu, "Refinements of two fractional versions of Hadamard inequalities for Caputo fractional derivatives and related results," Open Journal of Mathematical Sciences, vol. 5, no. 1, pp. 1-10, 2021.

[25] M. Z. Sarikaya, E. Set, H. Yaldiz, and N. Basak, "HermiteHadamard's inequalities for fractional integrals and related fractional inequalities," Mathematical and Computer Modelling, vol. 57, no. 9-10, pp. 2403-2407, 2013.

[26] M. Z. Sarikaya and H. Yildirim, "On Hermite-Hadamard type inequalities for Riemann-Liouville fractional integrals," Miskolc Mathematical Notes, vol. 17, no. 2, pp. 1049-1059, 2016.

[27] P. O. Mohammed, T. Abdeljawad, D. Baleanu, A. Kashuri, F. Hamasalh, and P. Agarwal, "New fractional inequalities of Hermite-Hadamard type involving the incomplete gamma functions," Journal of Inequalities and Applications, vol. 2020, Article ID 263, 2020. 